\title{
LAURA BATTISTA, UNA DRAMMATURGA DELLA PATRIA (1845-1884)
}

\author{
LAURA BATTISTA, A DRAMATIST OF THE HOMELAND (1845-1884)
}

Caterina Benelli

\section{Riassunto:}

Il presente lavoro è dedicato alla ricostruzione della significativa vicenda biografica e letteraria di Laura Battista, una dimenticata patriota e drammaturga lucana che opera nella seconda metà del XIX secolo. Nello specifico, si approfondirà il notevole contributo che la donna diede al risorgimento meridionale italiano attraverso l'analisi del dramma Emmanuele De Deo del 1869, intitolato alla memoria di un giovane martire giacobino che dimostra il protagonismo attivo, indissolubile e trasversale dell'autrice tra il versante teatrale e quello civile. Si evidenzierà l'impegno e il coraggio politico di una donna che, mediante il teatro, in tempi e luoghi ostili come quelli di una Basilicata patriarcale, filogovernativa e analfabeta, tenta di fronteggiare gli abusi politici del governo borbonico nel Mezzogiorno d'Italia e denunciare al contempo il destino di isolamento e subordinazione che la società riservava alla donna lucana ottocentesca.

\section{Parole chiave:}

Dramma storico; Risorgimento; Patriottismo rosa; Critica politica.

\section{Abstract:}

This papers aims to trace the biographical and literary legacy of Laura Battista, a forgotten Lucan patriot and dramatist. Specifically, it will examine the drama Emmanuel De Deo (1869), entitled in memory of a young Jacobin martyr. Her writings exude a critical approach to the patriarchal, pro-government and almost illiterate Basilicata; simultaneously airing the political abuse of the Bourbon government. A hostile atmosphere for the vindications of the Lucan women. Through her works, it will be highlighted the political courage of a strong woman.

\section{KEY WORD:}

Historical drama; Risorgimento; Gender nationalism; Political criticism. 


\section{L'OSSESSIVO MITO DELLA PATRIA: DALLA POESIA AL TEATRO}

La difficoltà femminile di affermarsi nel campo letterario è da sempre risaputa e risulta ancora più evidente in aree del Mezzogiorno italiano dove pochissime erano le donne che agli inizi del XX secolo avevano la possibilità di accedere a studi superiori, vittime di una mentalità patriarcale che le relegavano ad un compito meramente demografico e domestico. In tal senso appare ancor più meritevole ed oggetto di studio, la vicenda biografica e letteraria di Laura Battista, una donna lucana che operò in una regione caratterizzata da un isolamento geografico e da un ritardo culturale che ostacolavano qualsiasi tentativo di produzione letteraria. ${ }^{1}$ Inoltre, è ammirabile il coraggio politico e la passione civile che, come si analizzerà, ispirano e caratterizzano l'intera traiettoria letteraria di Laura Battista che si sviluppa in epoca risorgimentale.

opportuno sottolineare che la poetessa e drammaturga lucana, nata a Potenza nel $1845^{2}$ vive in realtà solo gli ultimi anni del Risorgimento italiano. Tuttavia, ancor prima dello sbarco di Garibaldi sul continente, l'animo della adolescente Battista viene scosso dall'esaltante piano insurrezionale lucano che ebbe luogo tra il tredici ed il diciotto agosto del 1860 con una serie di manifestazioni antiborboniche e filo unitarie in vari centri della provincia potentina (Lerra, 2008: 13). Il clima politico rivoluzionario che respira negli ambienti della borghesia potentina e la formazione umanistica inculcatagli dal padre Raffaele Battista, insegnante di latino e greco presso il Liceo classico di Potenza risultano elementi decisivi e centrali della poetica della giovanissima Laura.

Probabilmente, anche le tribolate e sfortunate vicende familiari quali la perdita prematura della madre e soprattutto di ben quattro dei cinque figli avuti dalla relazione con il nobile ma scioperato marito, Luigi Lizzardi, trovano ampia eco nelle pagine letterarie, intrise di profondo dolore ma al contempo di una ferrea speranza di riscatto personale e nazionale. In tal modo, l'intreccio bio-bibliografico si estrinseca in maniera lampante nel corso della sua breve esistenza; vita personale, vicende nazionali ed opere sono legate da un rapporto di causa ed effetto indisgiungibile.

Sin da bambina, nel salotto della sua casa potentina, ha l'opportunità di familiarizzare con le opinioni dei diversi liberali locali, pronti e motivati a sostenere i fermenti rivoluzionari contro il governo spagnolo. ${ }^{3}$ In particolare, risulta cruciale l'orientamento anti-borbonico paterno; al riguardo giova ricordare che il padre nel 1850 viene espulso

1 Per un quadro generale sulla situazione e sul grave ritardo culturale della Basilicata e le relative conseguenze sulla vita civile lucana, si veda Zanotti-Bianco (1926).

2 La data di nascita di Laura Battista è stata ricostruita in base all'atto di nascita conservato presso l'Ufficio di Stato Civile del Comune di Potenza. Dall'estratto dell'atto di nascita non ci sono altre annotazioni.

3 Per una esaustiva panoramica sul periodo borbonico in Basilicata si veda Verrastro (2011) dove si sottolineano le numerose azioni rivoltose contro i Borbone, organizzate nell'area lucana mediante un filone d'associazionismo politico che guardava ad un Mezzogiorno d'Italia libero ed indipendente da qualsiasi dominazione straniera. dal Real Collegio di Basilicata e fu diffidato dal pubblicare la traduzione di alcune opere di Seneca e, solo dopo l'Unità d'Italia e, dunque la scomparsa del regime borbonico, può riprendere la sua attività di intellettuale. Lo storico e critico letterario Giovanni Caserta, tra i pochissimi studiosi dell'opera letteraria della Battista, ci offre una motivazione originale ma plausibile sull'ossessione del padre che avrebbe puntato ed investito gran parte delle sue ambizioni e frustrazioni sulle qualità intellettuali della figlia, esercendo deliberate pressioni sulla stessa (Caserta, 2005: 9).

Così, i suoi esordi lirici sono innescati da acute e sentite riflessioni circa le complesse questioni risorgimentali meridionali come le disumane condizioni di povertà della gente lucana in cui sembrano fondersi i sogni e le speranze della Battista. Non appena viene proclamata l'Unità d'Italia, l'autrice pubblica una lunga poesia di evidente stampo patriottico intitolata Il 2 giugno 1861 per la festa nazionale commemorativa dell'Unità d'Italia e dello Statuto del Regno con cui comunica i suoi accesi sentimenti liberali e civili, esaltando le gesta di Garibaldi, del re Vittorio Emanuele e di tutte le popolazioni, che, coraggiosamente erano insorte contro lo straniero. Nel 1874 sono invece le necessità economiche che portano la Battista ad insegnare nel Convitto magistrale di Potenza; tuttavia per ragioni di salute e matrimoniali, pochi mesi dopo si trasferisce a Tricarico, un paese dell'entroterra materano ancora più interno e povero, dove la sua vita culturale viene messa a dura prova e non certo agevolata dalla convivenza con un marito ozioso, ricco e viziato, dedito al gioco e alle donne. Quest'ultimo, come se non bastasse, in pochissimi anni, incurante della sua cagionevole salute, non ebbe alcun riguardo facendole mettere al mondo ben cinque figli, dei quali, solo uno, l'unico maschio, riesce a sopravvivere. Tali tragedie umane, unite al suo immutato desiderio di modernizzare il Paese e favorire una coesione nazionale, fungono da stimolo permanente per la sua produzione letteraria. Di fatti, nel 1879, pur tra tanti dolori, con prefazione ed incoraggiamento di Abele Mancini $(1879)^{4}$, pubblica una prima raccolta di ottanta liriche intitolate leopardianamente i Canti che però non riscuotono il successo

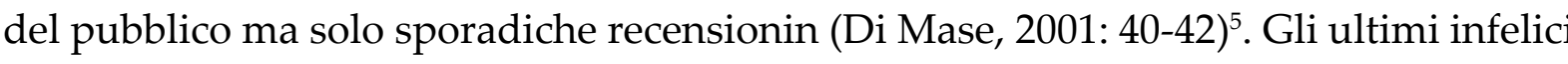
anni della sua vita li trascorre a Camerino dove le avevano assegnato una cattedra di lettere italiane. Purtroppo le sue condizioni di salute, già precarie, peggiorano tanto da indurla ad un forzato ritorno a Tricarico dove muore nell'agosto del 1884 all'età di trentotto anni.

Si potrebbe legittimamente pensare che la Battista considerasse la letteratura un efficace mezzo in cui trovare rifugio e consolazione e poter diffondere i suoi

4 Abele Mancini fu un acuto storico e poeta nato a Melfi nel 1846, apprezzato da Matilde Serao e sostenitore oltre che amico del suo corregionale Giustino Fortunato. Inoltre partecipò attivamente a moti risorgimentali del 1860, meritandosi in tal modo la stima della giovanissima Battista.

5 Recensioni circa la raccolta lirica della Battista si registrano sul Pungolo (aprile 1880) e sulla Nuova Antologia (maggio 1880).

Revista Internacional de Culturas y Literaturas, octubre 2016

ISSN: 1885-3625 
nobili ideali patriottici e civili. Come conseguenza, tanto le sue liriche come il suo dramma storico Emmanuele De Deo (Battista, 1869), scritto nel 1869, in tale prospettiva, sono il fedele riflesso e ricettacolo delle sue idee e dei suoi sentimenti, ricoprendo la letteratura di una funzione che è al contempo consolatrice e civile. Indubbiamente Laura Battista eredita dal padre l'amore per la cultura e per le lettere; aveva letto Goethe, Foscolo, Manzoni e Leopardi che segnano il cammino poetico della lucana. Ma ad incoraggiare ed ispirare la sua opera fu anche e soprattutto il suo vivo ed accorato interesse civile e politico che corrobora il suo tangibile patriottismo, ancor più apprezzabile se si considera l'insulso e desolante ambiente regionale da cui proveniva la drammaturga.

Laura Battista, dunque, attraverso una letteratura impegnata, intrisa di valori ed ideali spiccatamene risorgimentali, sembra voler scuotere prime e condurre poi i suoi corregionali, tradizionalmente filo governativi a ribellarsi contro il potere borbonico, ${ }^{6}$ permettendo la penetrazione dei principi di libertà e giustizia promossi in precedenza dalla Rivoluzione francese. Va ricordato che ella vive in un periodo caratterizzato da importanti e sconvolgenti fatti storici, da cambiamenti radicali i cui effetti straordinari probabilmente alimentarono la passione della Battista che finisce spesso con il mitizzare fatti e personaggi che si staccano dalla loro effettiva dimensione quotidiana, ed affollano la sua opera poetica e teatrale che esiste innanzitutto come testimonianza documentale ma anche come sogno ed evasione idealizzante e quindi come occasione per esaltarsi.

\subsection{Il patriottismo rosa nel dramma storico "Emmanuele De Deo"}

Laura Battista è senz'altro una donna lucana atipica in quanto, in una Basilicata patriarcale, fu protagonista non solo sul versante poetico ma anche su quello sociale partecipando attivamente alla vita culturale e politica del tempo, fatto oltremodo rilevante se si considera il ruolo, eufemisticamente secondario e meramente domestico che le donne ricoprivano. Il Risorgimento italiano, i personaggi come Cavour, Vittorio Emanuele, Garibaldi, l'Unità d'Italia generarono sentimenti profondi e radicali che spinsero la Battista alla rivendicazione del Paese natio e soprattutto a maturare un'idea di politica che lontana da accordi e strategici patteggiamenti, consente il recupero o la formazione di una patria civile in cui gli uomini, senza compromessi lottano per ottenere maggiore giustizia ed indipendenza. Forse, adesso risulta più agevole

6 Per una minuziosa contestualizzazione storica circa l'atteggiamento dominante e generale lucano nei confronti dei Borbone, si veda un articolo di A. Lerra (2014: 31), Presidente delle Deputazione di Storia Patria per la Basilicata e Professore ordinario di Storia Moderna presso l'Università degli Studi di Basilicata. comprendere perché l'autrice potentina abbia scritto un dramma storico, appena ventitreenne intitolato Emmanuele De Deo che ebbe notevole successo nel 1869.?

L'obiettivo principale della Battista sembra innanzitutto ricordare l'integrità morale e l'eroismo di De Deo, giovane studente che fu giustiziato dai Borboni perché promotore della rivolta giacobina napoletana del 1794. Il dramma composto di tre atti, in realtà se da un lato riflette in modo esemplare alcuni ideali della Battista, personificati dal De Deo, primo martire risorgimentale, dall'altro risponde probabilmente a delle precise pressioni familiari giacché bisogna sottolineare che lo sfortunato protagonista era un lontano parente del marito. Nello specifico, sarebbe stata Rosalba De Deo, moglie di Francesco Lizzardi e lontana discendente del martire De Deo, destinata a diventare suocera della Battista ad invogliare quest'ultima a scrivere il dramma. In realtà, indipendentemente dall'esegesi e dalle possibili interpretazioni e giustificazioni che possono motivare la realizzazione del dramma, resta l'originalità ed il vigore con cui una donna, benché lontana da ambienti teatrali rilevanti, abbia saputo costruire un'opera coraggiosa ed innovativa, considerando anche l'apatico contesto socio-culturale ottocentesco lucano. Infatti, una patriota convinta quale era Laura Battista non poteva di certo sorvolare su uno dei tragici ed emblematici momenti della storia meridionale italiana come il martirio di Emmanuele De Deo, protagonista indiscusso dell'omonimo dramma e nella realtà, attivo ed intrepido nemico del governo borbonico. In sintesi, giova ricordare che il De Deo, appena ventenne, nel maggio del 1793, ${ }^{8}$ durante un pranzo liberale presso Gioia del Colle (una località della provincia barese), si distinse per le numerose e corrosive satire pronunciate contro il re Ferdinando e la regina Maria Carolina e con un coltello minacciò la effigie del primo. Qualche mese dopo, i capi rivoluzionari, tra cui De Deo pensarono di stampare occultamente e diffondere la "Dichiarazione dei diritti dell'uomo" insieme con la "Costituzione francese". Emmanuele De Deo, secondo accurate ricostruzioni storicheavrebbe introdotto una copia nella reggia, facendola posare sul tavolo della regina Maria Carolina. A questo punto, la sorte del giovane precipita definitivamente quando un sacerdote, Pier Nicola Paterino, ${ }^{9}$ decide di denunciarlo e di conseguenza, a seguito di tale delazione, fece

7 Sulla notevole influenza esercitata dal clima familiare e dalla borghesia liberale rivoluzionaria potentina nei confronti del pensiero e dell'opera letteraria di Laura Battista, si veda l'interessante ritratto realizzato dalla studiosa e docente di Letteratura italiana presso l'Università degli Studi di Basilicata M. T. Imbriani (2000) contenuto in Appunti di letteratura lucana.

8 Per uno studio approfondito sulla figura e sulla storia del martire Emmanuele De Deo e la relativa congiura del 1794 è possibile consultare una vasta bibliografia. Per tutti si possono consultare T. Pedio (1976), e B. Croce (1953)

9 Un durissimo giudizio sul "vile e interessato tradimento" del sacerdote Pier Nicola Patarino è quello espresso dal docente ed economista pugliese G. Carano-Donvito in un articolo I De Deo in Gioia dal Colle pubblicato presso una rivista di storia ed arte dal nome Japigia, Società Editrice Tipografica, anno X, fascicolo I (gennaio-marzo), pp. 59-76. In questo articolo, nello specifico, lo studioso sottolinea come il sacerdote decide di denunciare il giovane studente ed i suoi amici per 
aprire un'inquisizione; così il nove maggio 1794 De Deo venne incarcerato insieme ad altri congiurati suoi amici, Galiani e Vitaliani che non a caso figurano nel dramma della Battista. Tuttavia il mito De Deo ${ }^{10}$ crebbe ulteriormente quando diede un'ennesima dimostrazione di assoluta fedeltà ai suoi compagni poiché, pur di non rivelare i loro nomi come colpevoli delle agitazioni antiborboniche prefer l' l'impiccagione e mantenne uno stoico silenzio persino quando fu strategicamente sottoposto a tortura sul patibolo nell'ottobre 1794

Si trattava, dunque, di un episodio troppo esemplare e commovente che la Battista riesce a trasformare in dramma storico in cui condensare i suoi furori patriottici e gli ideali etici. La scelta stessa del genere in questione è legata probabilmente alla ferma volontà dell'autrice di attenersi fondamentalmente alla verità effettiva, alla realtà storica e, al contempo, godere della relativa libertà artistica per personalizzare l'opera da un punto di vista tematico ma soprattutto stilistico e metodologico con cui risaltare determinati elementi in sintonia con il pensiero della Battista e stimolare una particolare reazione critica nello spettatore.

In definitiva, l'autrice lucana, opta per il dramma storico proprio perché garantirebbe una perfetta fusione tra storia e letteratura, tra il particolare ed il generale o se si preferisce tra aspetti e situazioni pubbliche e private. L'intenzione della Battista consiste nel offrire una esemplarizzazione specifica di un evento storico nazionale; a tale scopo si serve intelligentemente della figura di Emmanuele De Deo che si immola per i suoi amici e per la sua patria, diventando simbolo di perfezione comportamentale per il potenziale pubblico. Dunque, al suo coraggioso atto ad alla sua epica condotta si attribuisce una auspicabile esemplarità collettiva.

La maestria di Laura Battista risiede infatti nell'abilità con cui ritocca alcuni dati storici oggettivi, senza introdurre plateali menzogne, col fine di rendere totalmente credibile la rappresentazione, pur conferendo alla stessa una notevole simbolizzazione, plasmata attraverso l'interpretazione personale della drammaturga. Paradossalmente, proprio la minuziosa e a volte fin troppo eccessiva presenza di fatti storici datati e ormai passati rende il dramma anacronistico perché vincolato ad una realtà precisa, limitata e limitante. In altri termini, la Battista non ha saputo e probabilmente neanche voluto prescindere dal vero storico e dai suoi dettagli circostanziali, causando

scrupolo di coscienza al Ministro dell'epoca Acton. Quest'ultimo, di conseguenza, avrebbe passato subito i suoi ordini alla Polizia e precisamente al Commissario Caccia che intraprende le sue ricerche e perquisizioni che in ultima istanza, portano all'arresto di Emmanuele De Deo.

10 A conferma della fama del giovanissimo martire De Deo e dell'impatto che il suo sacrificio ebbe in buona parte del Mezzogiorno italiano si leggano le toccanti pagine in E. Striano, Il Resto di niente, Cava dei Tirreni, Avagliano, pp. 265-271. Inoltre, nel 1887 fu innalzato un monumento nella piazza principale del suo paese natale che gli ha dedicato un asilo per l'infanzia oltre che una strada situata nella parte antica dello stesso. Infine, fu omaggiato presso il Comune di Napoli (18 ottobre 1997) e all’Università degli Studi di Bari dove vi è una lapide con il suo nome, attualmente ubicata nel cortile della Facoltà di Giurisprudenza. involontariamente un precoce invecchiamento del suo dramma, che, al contrario, per essere atemporale o comunque più duraturo e universale necessita avvenimenti e coordinate storiche più generali. Ad esempio il truglio, ossia la delazione in cambio della commutazione della pena o della liberazione, perno attorno a cui ruota l'intero dramma, è tipico ed esclusivo dell'epoca e del sistema borbonico e quindi di comprensione immediata e facile da parte del pubblico di fine ottocento a cui doveva rivolgersi l'opera. È innegabile che la Battista all'avvenimento storico del De Deo, vi aggiunga una cospicua dose di eticità, dimostrando ad ogni modo che il suo dramma, benché edulcorato, in alcune scene e situazioni offre una complementare e coerente interpretazione della realtà effettiva senza contraddirla né tantomeno opporsi alla stessa.

Tuttavia, Laura Battista dimostra una certa originalità nel trattare fatti e personaggi locali, snobbando la tendenza generale del periodo che invece dava maggiore risalto a fenomeni e protagonisti europei o comunque di proiezione e caratura internazionale. L'autrice, inoltre, a dispetto del pensiero medievale che sopravvive ed impera nella produzione drammatica del XIX secolo, nel corso della sua opera dimostra un'ostinata e coraggiosa insofferenza politica che sfocia in lampante protesta verso il re che, ben lungi dall'essere considerato l'immagine terrena di Dio, si trasforma in uno dei bersagli preferiti dei personaggi, in quanto responsabile diretto o indiretto di ogni ingiustizia nel Regno di Napoli. L'opera della Battista la si potrebbe includere all'interno della tipologia del dramma storico proprio in virtù del suo deciso attacco al despotismo e della presenza di una severa critica politica e sociale. Si ricordi la netta denuncia realizzata dalla Marchesa Eleonora Fonseca Pimentel che si rivolge al padre del De Deo in questo modo: “I capi / Del governo son uomini, capaci / Quindi d'alte virtudi e rei delitti.../ [...] Ma quando, iniqui, abusano del loro / Grandissimo potere per farsi barbari / Oppressori dei popoli, di colpe / Inaudite bruttandosi, allora giusta / Dal petto dei magnanimi s'innalza / Una parola di vendetta al cielo"' (Battista, 1869: 35).

La Battista propende indubbiamente per il genere del dramma storico poiché desiderava ricostruire in maniera credibile lo spirito e l'atmosfera politica e civile del periodo pre-rivoluzionario. Ciò nonostante, l'autrice, come si è già accennato, riesce ad apportare e proiettare al contempo i suoi desideri patriottici personali, trasformando il dramma in una sorta di autorappresentazione mediante una trama specifica e la creazione letteraria di figure storiche, portavoci della sua cosmovisione. Insomma, la drammaturga potentina non inventa nulla né cerca di trasformare l'ideale in reale ma si accontenta di idealizzare il reale, partendo da eventi documentabili, rivolti ad un determinato pubblico, in un determinato momento. Una delle caratteristiche del dramma e dei dialoghi di cui si compone è l'enfasi e la retorica, fin troppo eccessiva con cui si esprimono sentimenti e concetti, benché sia stato concepito e scritto dopo il 
ragguardevole lasso temporale di settant'anni, rispetto agli avvenimenti a cui si riferisce. La Battista sembra smentire quella convinzione secondo cui la distanza consente una riflessione più pacata, serena ed imparziale di un fatto storico, adoperando criteri e filtri più obiettivi e razionali. Infatti, la drammaturga, in tal senso, forse perché implicata personalmente in rivolte e lotte preunitarie e antiborboniche che gli dovettero ricordare i moti insurrezionali giacobini napoletani del 1794, non è riuscita ad evitare un retoricismo smodato ed un coinvolgimento eufemisticamente appassionato. La Battista non stravolge affatto la storia ma utilizza una strategia letteraria, comunque legittima per la costruzione di un dramma, che, a sua volta, in quanto tale, non è tenuto a presentare pedissequamente materiali e risultati della ricerca storica con cui trasmettere il suo messaggio.

A livello tematico, il filo conduttore del dramma è rappresentato innanzitutto da una critica abbastanza esplicita verso la tirannia e, dunque, verso il malgoverno borbonico a cui corrisponde un parallelo eroismo, personificato dal giovane studente De Deo il quale, in nome della giustizia e della fedeltà verso i suoi amici, rifiuta di collaborare e scendere a patti con il vile e prepotente nemico, preferendo morire torturato ma libero e degno. Dunque, se da una parte si stigmatizzano gli eccessi, i vizi e gli errori della monarchia, dall'altra si esaltano i piccoli ma eroici gesti, di indiscusso valore morale ed evidente funzione edificante per il popolo lucano dell'epoca.

Il dramma risulta impregnato dal clima liberale che la Battista, già da fanciulla ha respirato in famiglia. Sin dalla prima scena del primo atto, l'autrice introduce quella esaltante ossessione che per lei rappresenta l'appartenenza alla patria e la successiva difesa della stessa come bene comune, esplicitata dalle retoriche domande che la Marchesa Eleonora Fonseca Pimentel amante del De Deo, rivolge agli amici Vitaliani, Galiani, Fiorentini e Luigia San Felice. "Non siete tutti amici miei, devoti / Ad una causa e d'una febbre accesi? [...] / Tu ancor, Luigia, sei dolente? Hai forse / Un'altra cura oltre alla Patria? / Anch'io la benedico, se ti prese amore / D'una patriota ardente Itala donna amor non dee che cittadini Eroi!"' (Battista, 1869: 8).

Un altro tema, decisamente più originaleintrodotto dalla Battista concerne la presenza di una orgogliosa quanto coraggiosa voglia emancipativa femminile meridionale, ancor più meritevole poiché inserito in un contesto patriarcale con una mentalità provinciale che considerava la donna come angelo del focolare domestico, remissiva, incapace ed indegna di qualsiasi tentativo di lotta. Nello specifico, la drammaturga lucana si serve di esortazioni impersonali che fa pronunciare in modo veemente alla Marchesa Eleonora, ribellandosi indirettamente al complesso di inferiorità di cui soffrivano le donne ottocentesche per rivendicare una parità assoluta tra i sessi anche nell'uso delle armi con cui superare tale inaccettabile condizione subalterna. "Sorgiamo adorni del valore antico, / Sorgiam d'ira frementi e in viso alteri.../ Trafiggiam concordi il vil nemico; / Noi pur, donne, nutriam maschi pensieri.../ Altre di noi formin vessilli eletti: / Altre cingan le spade ai giovinetti!" (Battista, 1869: 10). In realtà, con l'espressione nutriam maschi pensieri, la Battista, scopre la volontà delle donne $\mathrm{d}$ essere come l'uomo in tutto e per tutto, e tuttavia, inconsciamente ne decreta la sua intrinseca debolezza, dando per scontato già in partenza che gesti e idee belligeranti appartenevano esclusivamente all'universo maschile. La seconda scena, invece, si apre con un interessante dialogo tra il padre Vincenzo De Deo ed il figlio in cui il primo, a mio giudizio, riunisce magistralmente alcune caratteristiche comportamentali che orientano l'attitudine tipica del lucano, considerato pragmatico, paziente e rassegnato anche davanti alle iniquità socio-politiche.

O figlio, a che mi riducesti! [...] / T'adopri per inutil chimera, o figliuol mio! Sempre la forza alla ragion prevalse / Dalle più tardi etadi, e a noi non resta/ Che obbedir sempre e non dolerci mai! / Tu solo, Emmanuele, osi far motto / Dei diritti dell'uomo: osi chiamare/ Tiranno il rege, popolo la plebe... (Battista, 1869: 12).

Infatti, il padre sfodera tutta la sua saggezza e moderazione per mettere in guardia il figlio Emmanuele, troppo idealista e desideroso di gloria, presagendo un pericolo futuro. In realtà, la Battista sembra ricostruire la tipica dicotomia letteraria tra un padre razionale, prudente che preferisce la sicura mediocrità al glorioso rischio, ed un figlio spavaldo e istintivo, benché animato da nobilissimi propositi. La figura del padre, presente costantemente nel corso del dramma, sia pur implicitamente, si rivela fondamentale anche per comprendere ed approfondire la misoginia dilagante delle aree meridionali italiane. “Desiderio di gloria e cieca fede / Nella tua Leonora, in quella donna / O troppo presto o troppo tardi nata, Ti menano, o fanciullo, e Dio nol voglia! / Ad un sentir funesto! Emmanuele, Emmanuel, perdono a' tuoi deliri / Ma deh! Ti salva: la fatal amante / Non ascoltar, per Dio!" (Battista, 1869: 13).

Nello specifico, Vincenzo De Deo accusa il figlio di essere vittima delle cattive amicizie ma soprattutto della donna amata, ossia la Eleonora Pimentel, rea di rifiutare il suo ruolo domestico di moglie e madre esemplare e di dedicarsi alla vita attiva, inculcando idee sovversive e rivoluzionarie nella mente dell'ingenuo Emmanuele. Nel dramma della Battista si ripresenta dunque quella classica opposizione generazionale che tanto successo ha conosciuto nel teatro di fine cinquecento con un padre vecchio ed esperto della vita; quest'ultimo, inoltre lamenta l'inutilità della sua opera educativa verso un figlio intrepido al quale vanamente aveva insegnato il rispetto delle leggi e che invece, decide di sposare le nuove e liberali idee con l'entusiasmo e la generosità dei suoi giovani anni e del suo temperamento, insofferente verso ogni compromesso. È interessante notare come l'atteggiamento misogino paterno non solo non trova riscontro nelle repliche del figlio ma, al contrario, il giovanissimo Emmanuele, rispecchiando pienamente il pensiero femminista della Battista, esalta sperticatamente 
le virtù intellettuali ed umane della sua donna che ha avuto il merito di far accrescere e rinsaldare i più nobili valori, fra cui spicca il sentito patriottismo. Dunque, a differenza dell'anziano padre, lontano dal considerare la donna come origine di tutte le sciagure personali, lo studente sembra benedire il potere femminile, poiché proprio la Pimentel ha saputo potenziare le sue qualità morali ed attitudinali e fortificare gli ideali patriottici di lealtà, giustizia e libertà, raramente riscontrabili nel popolo ignorante, amorfo che, anzi, spesso stava dalla parte dei più forti. L'immagine della donna che ci presenta l'autrice è inequivocabilmente positiva, in quanto portatrice di valori che nobilitano e dignificano l'individuo e la società e quindi capace di far germogliare i pregi latenti presenti nell'uomo. A stravolgere ulteriormente lo stereotipo femminile in voga nella letteratura lucana ottocentesca, il dramma della Battista costruisce un atipico profilo della Marchesa Eleonora Pimentel, in cui accanto all'aspetto contemplativo coesiste alla perfezione l'aspetto attivo. A tal riguardo è significativa la quarta scena del primo atto allorché la Marchesa scopre che il suo amato Emmanuele era chiuso in prigione. “De Deo dicesti? Oh santi numi! Oh tristo / Sogno di ieri notte! Oh sposo mio, / Tu dunque in ceppi, ed io libera e viva? / Ma volerò a soccorrerti, l'estrema / Goccia del sangue mio spargerò lieta". (Battista, 1869: 23)

La Battista pare riversare tutto il suo pensiero e il suo ideale di donna battagliera, risoluta ed impavida, tutt'altro che passiva e rassegnata, attraverso la impulsiva reazione verbale della Marchesa, disposta a lottare audacemente pur di salvare la vita del suo uomo. Dipinge un ritratto femminile innovativo, rivoluzionario in quanto energico, volitivo e reattivo, distante dal tempo e dalla società in cui si inserisce la sua opera teatrale.

Un altro elemento centrale del dramma che a volte rappresenta un limite, è quello educativo o pedagogico. La Battista, infatti, attraverso le sventure del De Deo e dei suoi amici desidera sensibilizzare la gioventù napoletana e meridionale circa i pericoli e gli abusi dei governi tirannici per evitare di incappare nel futuro negli stessi errori e generare inique situazioni. Tale finalità è sinteticamente espressa dall'esternazione di Galiani, uno degli imprigionati, ai suoi amici di sventura: "Se a lungo in carcer resteremo, un libro/ Scriverem di memorie sulle nostre / Giovanili sventure: io così penso. / La gioventù partenopea leggendo / Quelle pagine allora a fieri sensi / Educherassi, e gioverà l'esempio che ne dite, o miei cari?" (Battista, 1869: 29).

Le parole di Galiani, inoltre, rimandano probabilmente all'idea di teatro e più in generale di letteratura della Battista la cui funzione per eccellenza consisterebbe nel formare generazioni future e nel fomentare la memoria storica collettiva, base imprescindibile per diffondere cultura, civismo e moralità. L'autrice, in definitiva, con il suo dramma encomiastico nei confronti della figura del giovane De Deo, si sofferma su un momento delicato della storia risorgimentale meridionale, ricostruendo il passato alla luce della sua visione socio-politica. Rievoca la minaccia della tortura, una pratica molto diffusa dal governo borbonico per ricevere informazioni d'ogni tipo sui dissidenti. Per questo, l'opera della Battista possiede anche un valore documentale da tramandare ai suoi corregionali, spesso ignari della realtà dei fatti o comunque inclini al facile oblio. La drammaturga, infatti, con tale opera crea una preziosa fonte di informazione, di rilevanza storica, pur cadendo nel proselitismo propagandistico in quanto si schiera nettamente a favore degli insorti giacobini, disprezzando apertamente la politica dei Borbone.

Infine, il terzo ed ultimo atto evidenzia l'incorruttibile lealtà del giovane studente Emmanuele che rifiuta qualsiasi patto opportunistico che gli avrebbe salvato la vita, optando perl'onore e la fedeltà versola patria e versogli amici. Di conseguenza, risultano vane le intercessioni e le mediazioni del padre prima, che lo spinge a rivelare anche un solo nome giacobino per ottenere la liberazione, e dell'amata che in ultima istanza, in un finale degno del miglior dramma romantico è disposta a fingersi una congiurata pur di salvare il suo Emmanuele. Quest'ultimo, offre una ennesima dimostrazione di integerrima eticità quando rifiuta in extremis una lettera contenente il foglio di grazia offerto dal re Fernando che sperava fino all'ultimo di strappargli i nomi di altri congiurati, ridandogli la libertà, peraltro acclamata a gran voce dal tumulto popolare che precedeva l'imminente e crudele esecuzione sul patibolo di Piazza Mercato di Napoli. Il protagonista era ormai deciso ad affrontare una morte stoica in grado magari di responsabilizzare quel popolo apatico e sempliciotto, fungendo da pungolo per una auspicata riscossa sociale, civile e politica. De Deo, tenta di consolare e convincere il padre sul suo eroico sacrificio, convinto che il popolo saprà ereditare ed applicare in futuro quelle virtù metatemporali che stava personificando quali l'onestà, il senso di giustizia, la dignità e la decenza: “La mia speranza, infruttuoso sangue / Non verserò da queste vene, e un giorno / Dal mio sepolcro esulterò vedendo / Nella polve i tirann abbominati..../ Popolo di Partenope [...] contempla come si muore. / Ad una ad una placida / L'anime nostre esaleremo.../ Aspirale Tu, popolo" (Battista, 1869: 46). Tutto il dramma, come si può constatare è costruito in maniera pedagogica con un'intenzione ossessivamente didattica e moralizzante, racchiusa perfettamente negli ultimi versi dello stesso e pronunciati dall'affranta ma orgogliosa Marchesa Eleonora De Fonseca Pimentel che promette di smettere di piangere solo il giorno in cui l'Italia saprà trarre insegnamento dal martirio del De Deo. Appare dunque abbastanza evidente la finalità che la Battista insegue nel momento in cui scrive la sua opera teatrale. L'autrice sembra concepirla per divulgare il sacrificio del De Deo affinché almeno la sua isolata Lucania ottocentesca, si scuota dal torpore civile e custodisca nella sua memoria collettiva il 
martirio del giovanissimo studente, ${ }^{11}$ traendone i dovuti insegnamenti e una doverosa presa di coscienza storica con cui avere consapevolezza della propria identità culturale. La Battista contribuisce a consolidare il mito di Emmanuele De Deo, coerente e leale fino all'ultimo, tanto da spiazzare e sconfiggere moralmente i Borbone e le loro navigate ed efficaci modalità intimidatorie e violente, stavolta nettamente rifiutate dall'intrepido studente che impone il suo spirito liberale e la sua razionalità.

Il dramma, è interpretabile anche come un'opera didattica che presenta una tesi, ovvero la potenziale corruttibilità dell'uomo, messo alla prova e la dimostra; conferisce dignità ed eroismo al martire che umilia eticamente la prepotenza borbonica, il suo abuso dispotico e illegale, preferendo ad una comoda ed opportunistica infamia l'integrità morale che poi significa fedeltà verso se stesso oltre che verso gli amici. Invece, un eroismo parziale, se non di pura facciata è quello dimostrato dalla Marchesa Eleonora, apparentemente disposta ad immolarsi ed autodenunciarsi davanti al re Fernando per salvare l'amato Emmanuele. In realtà è un gesto ipocrita poiché rappresenta un sacrificio strumentale e solo teorico, destinato ad immiserirsi visto che è la stessa donna che allude ad amicizie e raccomandazioni di personaggi influenti che avrebbero evitato guai seri.

Nel complesso, il dramma risponde tematicamente all'ideologia e alla formazione culturale liberale della Battista, al suo incrollabile e sincero amore di patria che la condusse ad enfatizzare la vicenda di De Deo. Tuttavia, il dramma, genere allora sconosciuto alla Battista, non riscosse particolare successo forse perché appariva come un'opera di indiscussa volontà e impegno ma priva di schiettezza, ispirazione, originalità e soprattutto perché di argomento ormai datato e anacronistico per un meridione in preda a nuove realistiche urgenze fra cui spicca la protesta contadina, il brigantaggio e la obbligata quanto drammatica emigrazione. L'autrice ha insistito troppo sul patriottismo e funzionalmente ad esso ha optato per un dramma in cui domina il dialogo, dove non si raggiunge mai la plasticità dell'azione. Inoltre, le numerose parti dialogate spesso peccano di freschezza verbale e risultano appesantite da una retorica stucchevole e di circostanza, rivolta ad una smodata esaltazione dell'idea di patria che finisce per offuscare i sentimenti più genuini ed umani, come ad esempio il dolore di un padre nel vedere un figlio prossimo alla tortura. Anche quando si produce l'incontro-scontro tra Vincenzo De Deo e l'ardita Eleonora sui valori da insegnare al figlio Emmanuele, manca tra i due un dialogo serrato, netto e vibrante; anzi, ognuno si limita a rispondere evasivamente all'altro, esponendo i principi di base

11 Può essere utile ed interessante segnalare come secondo lo scrittore Mario Benedetti, co-autore del saggio El teatro como guardián de la memoria colectiva, 2007 dedichi un intero capitolo (il secondo) alle finalità generali del teatro tra cui spicca proprio quella funzione di guardiano, applicabile perfettamente all'opera della Battista, capace di formare una memoria collettiva storica assolutamente necessaria affinché un popolo conosca ed apprenda da eventi passati. delle loro opposte filosofie esistenziali. D'altronde, non bisogna dimenticare che la tecnica di scrittura della Battista era derivata dalla pura e a volte meccanica imitazione di altri modelli letterari (Foscolo e Manzoni, Aleardi in poesia), per cui ne risulta uno stile poco personale. Infatti nel dramma, scritto in versi endecasillabi di buona fattura che confermano comunque una maestria versificatoria ben assimilata, è abbastanza frequente trovare un linguaggio aulico e ricercato, mutuato direttamente dai libri (Caserta, 1993: 253). Così, non deve meravigliare l'utilizzo di vocaboli desueti e arcaici, appartenenti ad una letteratura arcadica ed inadatti alla situazione drammatica. Si pensi alle forme verbali avria o saria, oppure ai sostantivi donzella o garzoni. In realtà, l'eccesso di retoricismo, il vigore espressivo, gli atteggiamenti di maniera che impregnano molte delle pagine dell'opera teatrale sono funzionali alla finalità ed al messaggio patriottico che desiderava veicolare.

In sintesi, l'autrice cerca di creare illusione, di conquistare e coinvolgere il lettore e/o spettatore tanto da farloidentificare conil personaggio principale con la sua problematica situazione e la sua tensione previa al martirio. Laura Battista, inoltre, come si è già sottolineato è stata una donna atipica, coraggiosa, combattiva che intenzionalmente ha trasferito la sua passione politica e civile, i suoi ideali risorgimentali italiani nel suo unico e dimenticato dramma ed ha indirettamente contribuito al processo emancipativo della donna lucana nella seconda metà del XIX secolo. La sua condizione di donna colta, politicamente preparata ed esposta è ancor più apprezzabile perché espresso in un contesto ideologicamente tradizionale e chiuso come quello della provincia potentina. Essa può e deve costituire l'emblema della presenza alla vita attiva ed intellettuale in una società lucana analfabeta e maschilista, segno dei tempi nuovi che avanzavano. Purtroppo, le qualità artistiche ed umane della patriota Laura Battista, a differenza del protagonista del suo dramma, quasi mai trovano spazio e risalto a livello storico e letterario, forse perché si preferisce ancora una femminilità più convenzionale e meno reattiva.

\section{RIFERIMENTI BIBLIOGRAFICI}

Battista. L., Emmanuele De Deo, dramma storico, Potenza, Santanello, 1869. Benedetti, M., El teatro como guardián de la memoria colectiva, Buenos Aires, Corregidor, 2007.

Croce, B., La Rivoluzione napoletana del 1799. Biografie, Racconti, Ricerche, Bari, Laterza, 1953.

Caravano Donvito, G., "I De Deo in Gioia dal Colle”, Japigia, anno X, fascicolo I (gennaio-marzo), pp. 59-76

Caserta, G., Lo spirito del silenzio, Scrittori lucani, Taranto, Scorpione, 1988.

----, Storia della letteratura lucana, Venosa, Osanna, 1993, p. 253. 
----, Laura Battista, Séguito dei Canti, Matera, Giannatelli, 2005, p. 9.

Di Mase, C., La biblioteca dei conti Gattini, Altrimedia, Matera, 2001, pp. 40-42.

Hubert, M. C., Le théâtre, Paris, Collins, 1988.

Imbriani, M. T., Appunti di letteratura lucana: ventisette ritratti d'autore: dal Medio evo giorni nostri, Potenza, Consiglio regionale della Basilicata, 2000, pp. 97-104.

----, "Intorno a Laura Battista: nuove poesie e documenti", Bollettino storico della Basilicata, Venosa, Osanna, n. 21, a. XX (2005), pp. 149-160.

Lerra, A., "Nel Mezzogiorno provinciale napoleonico", Bollettino storico della Basilicata, n. 24, a. XXIII (2008), p. 13.

----, La Basilicata per l'unità d'Italia, Ed. Guerini e Associati, Milano, 2014, p. 31.

Mancini, A., Canti di Laura Battista, Matera, Conti, 1879.

Palant, P., El texto dramático, Buenos Aires, Centro Editor de América Latina, 1968

Pedio. T., Massoni e giacobini nel Regno di Napoli. Emmanuele De Deo e la congiura del 1794, Matera, Montemurro, 1976.

Perrone, B., "Laura Battista”, Bollettino della biblioteca provinciale di Matera, nn. 2324, vol. XV, (1994), pp. 69-84.

Ribao Pereira, M., Textos y representación del drama histórico en el romanticismo español, Pamplona, Anejos Rilce, Eunsa, 1999.

Ripoli, F., "Laura Battista, poetessa della patria e del dolore”, Bollettino della biblioteca provinciale di Matera, a. VII, n. 12, 1986

Serpieri, A., Come comunica il teatro: dal testo alla scena, Milano, Il Formichiere, 1978. Spang, K., El drama histórico, teoría y comentarios, Pamplona, Eunsa, 1991.

Spinelli, T., Basilicata, Brescia, La Scuola, 1987.

----, Poeti lucani tra Ottocento e novecento, Francavilla sul Sinni, Capuano, 2000.

Striano, E., Il Resto di niente, Cava dei Tirreni, Avagliano, pp. 265-271.

Verrastro, V., La Basilicata per l'unità d'Italia: idealità, azione politica, istituzioni (17991861), Lagonegro, Zaccara, 2011, pp. 63-103

Zanotti-Bianco, U., La Basilicata, Roma, Collezione Meridionale Editrice, 1926. 